Research Article

\title{
Static and Dynamic Brazilian Tests on Layered Slate considering the Bedding Directivity
}

\author{
Xuefeng Ou $\mathbb{D}^{1},{ }^{1}$ Xuemin Zhang, ${ }^{2}$ Han Feng $\mathbb{D},{ }^{2}$ Cong Zhang, ${ }^{3}$ Xianshun Zhou, ${ }^{2}$ \\ and Lei Wang ${ }^{4}$ \\ ${ }^{1}$ National Engineering Laboratory of Highway Maintenance Technology, Changsha University of Science \& Technology, \\ Changsha 410114, Hunan, China \\ ${ }^{2}$ School of Civil Engineering, Central South University, Changsha, Hunan, China \\ ${ }^{3}$ School of Civil Engineering, Central South University of Forestry and Technology, Changsha, Hunan, China \\ ${ }^{4}$ School of Civil Engineering, Changsha University of Science \& Technology, Changsha 410114, Hunan, China \\ Correspondence should be addressed to Han Feng; fenghan_csu@163.com
}

Received 13 August 2020; Revised 16 October 2020; Accepted 3 December 2020; Published 16 December 2020

Academic Editor: Payam Shafigh

Copyright (c) 2020 Xuefeng Ou et al. This is an open access article distributed under the Creative Commons Attribution License, which permits unrestricted use, distribution, and reproduction in any medium, provided the original work is properly cited.

\begin{abstract}
A layered rock usually exhibits strong anisotropy due to its layered structure. In order to study the anisotropic effect on its static and dynamic tensile properties, a medium strength anisotropy slate is chosen and tested in five groups of bedding plane dip angles. The dynamic tests were carried out by a split Hopkinson pressure bar (SHPB), and the failure process of rock samples is recorded by a high-speed camera. The failure mode and strength characteristic of the slate are analyzed. The static test results show that layered structure significantly affects the failure mode, and the influence of the bedding plane depends on the degree of anisotropy. The static and dynamic "tensile strength" exhibit the " $U$ " type strength anisotropy. For samples in the same dip angle group, the "tensile strength" shows clear dynamic strengthening effect, and the growth rate is most significant at $\theta=45^{\circ}$.
\end{abstract}

\section{Introduction}

Due to the sedimentation and tectonic loading, many rocks like slate, phyllite, schist, and gneiss, naturally exhibit regular layered structures. These structures usually make the rock materials have one dominant direction of planar anisotropy. In contrast to a homogeneous rock mass, which has the same mechanical properties in all directions, the mechanical properties of a layered rock mass change with the change in bedding direction [1].

Meanwhile, tensile strength is one of the most important parameters of rock materials. Failure of brittle rock normally initiates from tensile fracture due to its extraordinary lower resistance to tension rather than to compression [2]. In many engineering applications, parameters such as the stability of the chamber excavation and hydraulic fracturing largely depend on the tensile strength of the rock $[3,4]$. Therefore, it is necessary to accurately determine the tensile properties of the layered rock mass.
Various methods have been proposed for measuring the tensile strength of rocks. Due to the difficulties associated with experimentation in direct tensile tests, indirect methods are widely used as the convenient alternatives to measure the tensile strength of rocks, such as the Brazilian disc test, the ring test, and the bending test $[5,6]$. Among these indirect test methods, Brazilian test, maybe the most popular one due to its features of convenient specimen preparation and experimental implementation. It has been recommended for testing tensile strength of rock materials by the International Society for Rock Mechanics (ISRM) in 1978 [7] and the American Society for Testing and Materials (ASTM) [8]. Therefore, Brazilian test is chosen in this study. Brazilian tests have been chosen by some researchers to investigate the tensile properties of layered rock [9], such as Tavallali A, André Vervoort on sandstone [10], Khanlari et al. on sandstone [11], and Lee and Pietruszczak on slate and schist [12]. 
Researchers also have extended the Brazilian test to the regime of dynamic testing [13]. The split Hopkinson pressure bar (SHPB) is widely adopted to conduct the dynamic Brazilian test to attain higher loading rates $[14,15]$. Dai and Xia conducted SHPB tests on Brazilian discs of Barre granite to measure the anisotropy dynamic tensile properties [5]. Qiu et al. measured the dynamic tensile properties of phyllite with the Brazilian test [16]. However, the dynamic tensile property of layered rock mass still received limited attention. It is thus necessary to investigate the static and dynamic anisotropy tensile properties considering the bedding directivity.

In this study, the static and dynamic tensile Brazilian tests were conducted by a material testing machine and split Hopkinson pressure bar (SHPB), respectively. Five groups of medium strength anisotropy slate samples are tested with different bedding dip angles $\left(\theta=0^{\circ}, 30^{\circ}, 45^{\circ}, 60^{\circ}\right.$, and $\left.90^{\circ}\right)$. The crack process in SHPB is recorded with a high-speed camera. Then, the static and dynamic tensile properties and failure modes are compared to investigate the effect of anisotropy on the tensile properties.

\section{Experimental Methods}

2.1. Static and Dynamic Testing Facilities. A SANS material testing machine is used to conduct the quasistatic tests, as shown in Figure 1. The machine's maximum static load is $\pm 100 \mathrm{kN}$, and the loading measurement accuracy is $\pm 0.5 \%$. The test used a $0.05 \mathrm{~mm} / \mathrm{min}$ constant loading rate for all samples. The "tensile strength" in the Brazilian test means the maximum tensile stress at the center of the disc sample when the sample lost its total bearing capacity. The term "tensile strength" is set into quotation marks, because pure tensile fracturing is observed only for special configurations depending on the foliation-loading angle. Within this article, independent on the observed fracture pattern, all evaluations are performed according to the classical equation for Brazilian tests, which assumes isotropic homogeneous material [17]:

$$
\sigma_{t}=\frac{2 P_{c}}{\pi D L}
$$

where $P_{c}$ is the maximum loading value, $D$ is the diameter of the sample, and $L$ is the thickness of the sample.

As shown in Figure 2, the dynamic test is conducted by using a $50 \mathrm{~mm}$ SHPB system comprising a $200 \mathrm{~mm}$ striker bar, a $1500 \mathrm{~mm}$ incident bar, and a $1200 \mathrm{~mm}$ transmission bar. All the bars are made of $40 \mathrm{Cr}$ alloy steel, with a Young's modulus of $240 \mathrm{GPa}$, a P-wave velocity of $5400 \mathrm{~m} / \mathrm{s}$, and a density of $7810 \mathrm{~kg} / \mathrm{m}^{3}$. A cone-shaped striker is used to generate a half-sine incident wave and provide constant strain rate loading until sample failure [18-21]. A Photron Fastcam SA1.1 high-speed camera is utilized to monitor the whole failure process of the samples. When the stress wave generated by the punch reaches the strain gauge of the incident rod, the high-speed camera linked with the oscilloscope is triggered and starts to record. The frequency band range of the ultradynamic strain tester is $0 \sim 1 \mathrm{MHz}$. The

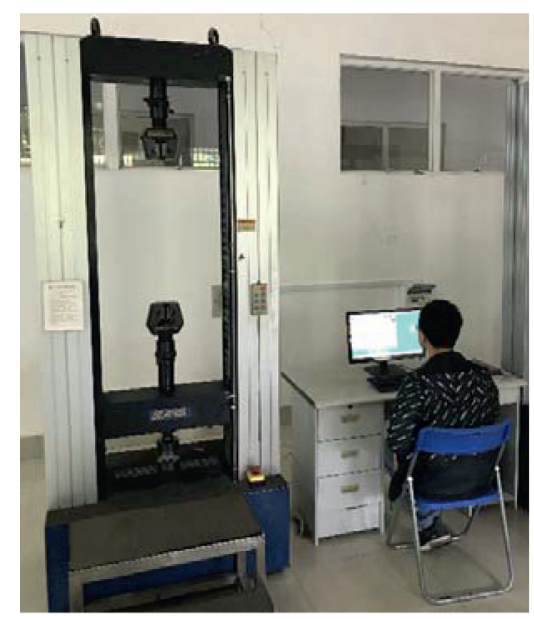

FIgURE 1: Static Brazilian test with the SANS material testing machine.

locations of the strain gauges mounted on the incident and transmitter bars are both $1 \mathrm{~m}$ away from the sample.

In the dynamic test, a rock sample is firstly placed between the incident bar and the transmission rod. Then, the gas pressure in the gas gun is set to $0.5 \mathrm{MPa}$. The valve is opened after the air pressure is stabilized; the gas pressure pushed the bullet to impact the incident bar. The uniform air pressure can make the punch produce constant impact velocity and obtain constant incident stress wave.

The stress pulse information, including the incident strain pulse $\varepsilon_{I}$, the reflected strain pulse $\varepsilon_{R}$, and the trsansmitted strain pulse $\varepsilon_{T}$, is collected by the strain gauges on the bars. Previously, studies show that two ends of the disc sample can reach the stress equilibrium state during the dynamic Brazilian test $[22,23]$. Therefore, the equilibrium hypothesis can be introduced into the analysis of the dynamic Brazilian splitting test result

$$
\varepsilon_{I}(t)+\varepsilon_{R}(t)=\varepsilon_{T}(t) .
$$

Thus,

$$
P(t)=E A \varepsilon_{T}(t),
$$

where $A$ is the cross-sectional area of the incident rod and the transmission rod (the diameter of the incident rod and the transmission rod is $d$ ), and $E$ is the elastic modulus of the incident rod and the transmission rod.

Thus, the dynamic tensile strength can be calculated using

$$
\sigma_{t}=\frac{2 P(t)_{\max }}{\pi D L},
$$

where $P(t)_{\max }$ is the maximum load on the samples during the dynamic Brazilian test.

The energy of the incident strain pulse is as follows:

$$
W_{I}=\left(A_{e} C_{e} E\right) \int \varepsilon_{I}(t)^{2} \mathrm{~d} t
$$

where $A_{\mathrm{e}}, C_{\mathrm{e}}$, and $E_{\mathrm{e}}$ are the cross-sectional area, the sonic velocity, and Young's modulus of the incident bar. 


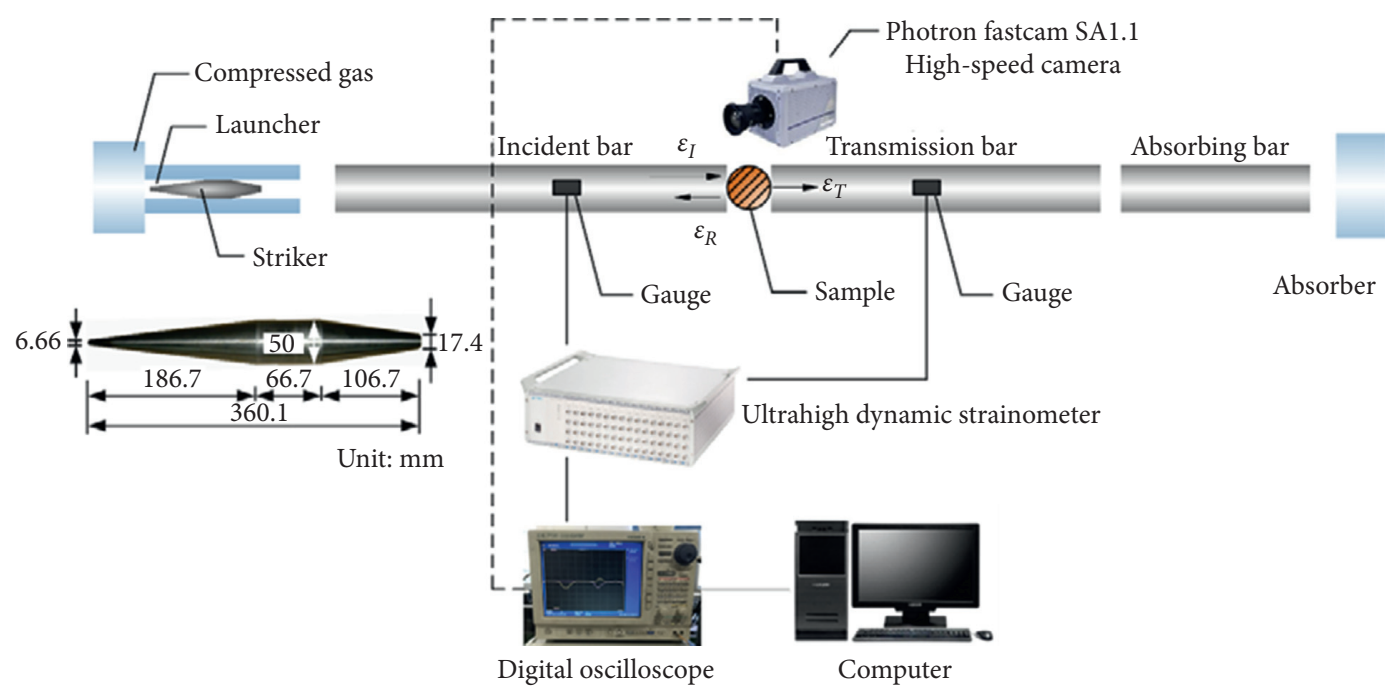

Figure 2: Dynamic Brazilian test with the SHPB system.

The energy of the reflected strain pulse is as follows:

$$
W_{R}=\left(A_{e} C_{e} E\right) \int \varepsilon_{R}(t)^{2} \mathrm{~d} t
$$

The energy of the transmitted strain pulse is as follows:

$$
W_{T}=\left(A_{e} C_{e} E\right) \int \varepsilon_{T}(t)^{2} \mathrm{~d} t .
$$

The energy absorbed by the rock sample [24] is as follows:

$$
W_{S}=W_{I}-W_{R}-W_{T}
$$

2.2. Brazilian Disc Preparation. A layered slate is selected to conduct the static and dynamic Brazilian test. In this test, five groups of samples which contain different dip angles $\theta$ (including $0^{\circ}, 30^{\circ}, 45^{\circ}, 60^{\circ}$, and $90^{\circ}$ ) are prepared with a diameter of $50 \mathrm{~mm}$ and a thickness of $25 \mathrm{~mm}$, where the dip angle $\theta$ is the angle between the orientation of layers and the horizontal plane, as shown in Figure 3. In order to ensure the reliability of the test results, the nonmetal ultrasonic detector is used to test the wave velocity $v_{p}$ of the samples. The samples with large difference in $v_{\mathrm{p}}$ have been eliminated before the tests. Three samples are prepared for each specific dip angle.

\section{Geological and Mechanical Characteristics of the Slate}

The studied slate was collected from Jiangxi Province, China. A chemical composition analysis of the slate yielded the following composition: $59.05 \% \mathrm{SiO}_{2}, 18.56 \%$ $\mathrm{Al}_{2} \mathrm{O}_{3}, 6.87 \% \mathrm{Fe}_{2} \mathrm{O}_{3}, 0.24 \% \mathrm{CaO}, 1.84 \% \mathrm{MgO}, 3.47 \% \mathrm{~K}_{2} \mathrm{O}$, and $2.03 \% \mathrm{Na}_{2} \mathrm{O}$. The slate is mainly composed of quartz, chlorite, mica, feldspar, and dark minerals. This slate is banded in appearance, and the bands have a width of $0.3 \sim 0.7 \mathrm{~mm}$.
Before the dynamic test, the uniaxial compressive strength (UCS) (Table 1) of the slate was measured in a uniaxial compression test with an Instron 1346. The samples are prepared with a diameter of $50 \mathrm{~mm}$ and a height of $100 \mathrm{~mm}$. The loading rate of the uniaxial compression test is $0.5 \mathrm{MPa} / \mathrm{s}$.

Referring to the definitions of Ramamurthy, the anisotropy ratio $\left(R_{\mathrm{c}}\right)$ equation for transversely isotropic materials is as follows [25]:

$$
R_{c}=\frac{\sigma_{c i(0)}}{\sigma_{c i(\min )}}
$$

where $\sigma_{c i(\min )}$ is the minimum compressive strength obtained at different angles and $\sigma_{c i(0)}$ is the minimum compressive strength of $0^{\circ}$.

According to the calculation results of formula (9), the rock anisotropy can be determined by Table 2 .

When the strength parameters in Table 1 are substituted into equation (2), the resulting value is $R_{c}=2.42$. Therefore, the slate can be classified as a rock with medium anisotropy.

\section{Testing Results of Static Tensile Properties}

Figure 4 shows the failure modes of slate samples with different bedding dip angles under the Brazilian test. However, the failure modes present in Figure 4 show that samples under the bedding angle have not failed along the loading direction, except for the specimen with bedding angle of $\theta=90^{\circ}$. Due to the influence of bedding plane, there are three failure modes of slate samples: (1) along bedding failure $\left(\theta=45^{\circ}\right.$ and $\left.\theta=90^{\circ}\right)$; (2) nonbedding failure $\left(\theta=0^{\circ}\right.$ and $\left.\theta=30^{\circ}\right)$; and (3) mixed failure along bedding plane and nonbedding plane $\left(\theta=60^{\circ}\right)$.

Figure 5 shows the distribution curve of "tensile strength" of slate with different bedding dip angles. Figure 5 shows that the "tensile strength" decreases first and then increases with the increase of bedding angle. It indicates that the bedding plane has a significant influence on the "tensile strength." The variation trend of "tensile strength" with 


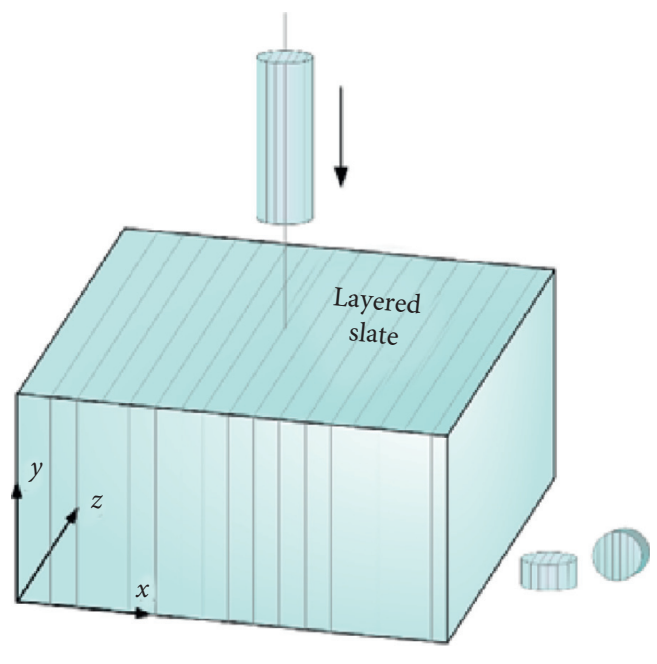

(a)

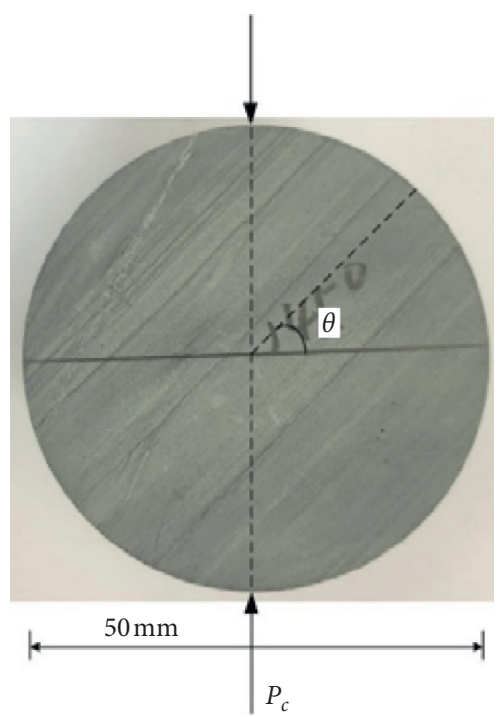

(b)

FiguRe 3: Brazilian test sample preparation.

TABLE 1: Uniaxial compressive strength.

\begin{tabular}{lccccc}
\hline$\theta$ & $0^{\circ}$ & $30^{\circ}$ & $45^{\circ}$ & $60^{\circ}$ & $90^{\circ}$ \\
\hline$U C S(\mathrm{MPa})$ & 167.29 & 79.29 & 73.31 & 69.01 & 147.25 \\
\hline
\end{tabular}

TABLE 2: Classification of transversely isotropic materials based on the strength anisotropy parameters.

\begin{tabular}{lc}
\hline Range & Class \\
\hline $1.0<R_{c} \leq 1.1$ & Semi-isotropic \\
$1.1<R_{c} \leq 2.0$ & Low anisotropy \\
$2.0<R_{c} \leq 4.0$ & Medium anisotropy \\
$4.0<R_{c} \leq 6.0$ & High anisotropy \\
$6.0<R_{c}$ & Extremely high anisotropy \\
\hline
\end{tabular}

bedding dip angle is like that of static uniaxial compressive strength, which also belongs to a " $U$ " type anisotropic curve. The difference between them is that the characteristic value of "tensile strength" is more significant, which is $\sigma_{t(0)} / \sigma_{t(90)}=2.07$ and $\sigma_{t(0)} / \sigma_{t(\min )}=3.52$. Additionally, the minimum value of "tensile strength" $\sigma_{t(\min )}$ is located at $\theta=45^{\circ}$, while the minimum value of uniaxial "tensile strength" is located at $\theta=60^{\circ}$.

\section{Dynamic Tensile Properties of the Slate}

Figure 6 shows the typical stress wave curves collected on the incident bar and transmission bar in the dynamic Brazilian test of the layered slate. According to the ISRM recommended method, the stress balance between the two ends of the sample is required to ensure the reliability of the test.

Figure 7(a)-7(e) shows the stress impulses at both ends of the samples with different dip angles. The (In $+\mathrm{Re})$ curve plots the incident stress impulse plus the reflected stress impulse and is approximately equal to the transmitted stress impulse curve, indicating that a stress equilibrium is achieved during the loading process, further verifying the validity of the test results. Also, it means the bedding planes have little effect on the stress balance of the samples in the dynamic Brazilian test.

5.1. Dynamic Failure Mode of the Slate. Figure 8 shows the failure process of specimens in the dynamic Brazilian test under different dip angles. According to the relationship between crack development and bedding planes, the failure modes of specimens can be divided into four types: (1) tensile splitting failure along bedding; (2) sliding failure along bedding; (3) tensile splitting failure across bedding; and (4) composite failure mode along and through bedding simultaneously. At the same time, it can be seen from the figure that the failure modes of samples under different dip angles are different. Therefore, the failure mode and cracking process are summarized as follows:

The whole cracking process of all samples can be concluded as follows: the impact load just reaches the left end of the sample; the sample begins to crack; the main crack develops, and the sample finally fails.

When the sample is $\theta=0^{\circ}$, the loading direction is perpendicular to the bedding plane. At this time, the specimen is destroyed through the bedding plane at both ends of the loading. Both ends of the loading crack almost at the same time are spread through the edge of the specimen. There is no through crack between the two ends of the specimen, only the local failure near the loading end.

When the sample is $\theta=30^{\circ}$, the crack mainly develops along the bedding plane to the edge of the specimen at the loading end. At the other end, the crack develops perpendicular to the bedding plane at first, and then, it turns to develop along the bedding direction. Finally, the crack develops downward perpendicular to the bedding plane, the crack is S-shaped and it connects both ends of the sample. 


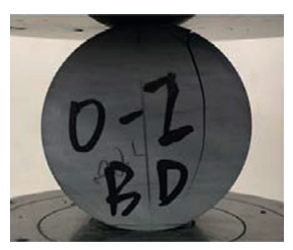

(a)

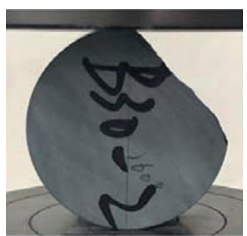

(b)

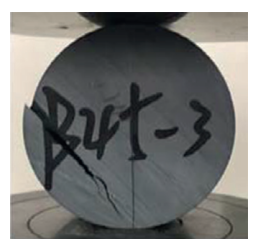

(c)

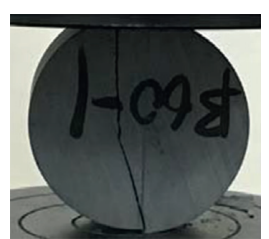

(d)

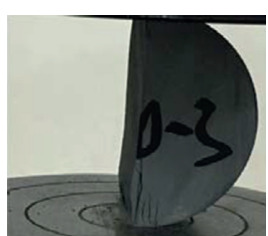

(e)

FIgURE 4: Failure mode of slate in the Brazilian test.

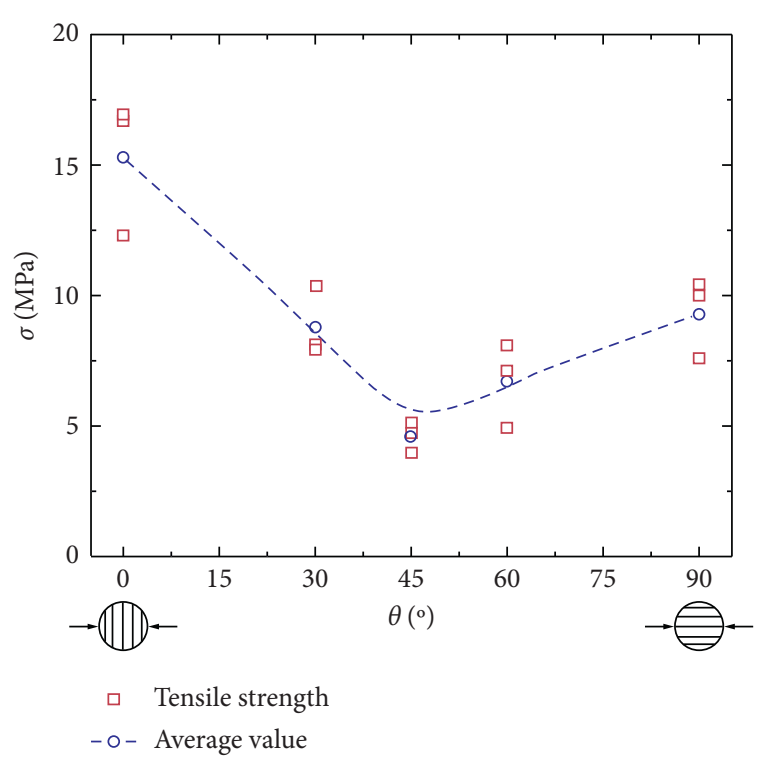

FIgURE 5: "Tensile strength" curve of the layered slate with different dip angles.



FIgURE 6: Typical stress wave during the dynamic Brazilian test.

When the specimen is $\theta=45^{\circ}$ and $\theta=60^{\circ}$, the sample is mainly failed along the bedding plane. In the process of dynamic loading, cracks along the bedding plane appear at the loading end of the sample, and the sample is destroyed rapidly. There is only one main crack along the bedding plane under the dip angle.

When the specimen is $\theta=90^{\circ}$, the loading direction is parallel to the bedding plane of the specimen. The failure process of the specimen is cracking along the bedding plane, and the failure mode is typical tensile splitting failure along the bedding plane. Under this bedding angle, all cracks are along the bedding plane. During the loading process, the main crack firstly appears at the center of the sample and then develops at the both ends.

Figure 9 shows the ratio of crack length along bedding to total crack length under each dip angle. The ratio increases rapidly with the increase of the bedding angle and only slightly decreases when it is $60^{\circ}$ to $90^{\circ}$. With the increase of the bedding angle, the influence of the bedding surface on the impact splitting failure of the specimen is prominent, especially when it rises to $60^{\circ}$, and the specimen is mainly sliding along the bedding plane, which is generally a single crack. However, during the crack development process of $90^{\circ}$, some inclined cracks appear at both ends of the specimen, which leads to a decrease in the ratio.

5.2. Dynamic “Tensile Strength" Characteristic. Table 3 lists the measured dynamic "tensile strength" of the layered slate under each dip angle, including the loading rate [26] and energy absorption. The average "tensile strength" varies with dip angles, while the energy absorption is also varying with dip angles. The energy absorption value represents the new surface area of cracks generated during the dynamic test [27]. The energy absorption of slate is highest when $\theta=30^{\circ}$ and the lowest at $\theta=45^{\circ}$ under conventional impact Brazilian splitting. The average value of dynamic "tensile strength" at each bedding dip angle is presented in Figure 10. The results show that the dynamic average "tensile strength" decreases first and then increases with the increase of the dip angle. It is also a typical U-type anisotropic curve. The maximum and minimum values of average dynamic "tensile strength" appear at $\theta=0^{\circ}$ and $\theta=60^{\circ}$, respectively.

\section{Discussion}

6.1. Static Brazilian Test. The fracture toughness anisotropy coefficient is adopted to evaluate the effect of rock anisotropy on the failure mode of the Brazilian disc sample [28]. The anisotropy degree of splitting strength $\alpha_{\mathrm{t}}$ is defined as the ratio of the maximum "tensile strength" to the minimum "tensile strength",

$$
\sigma_{t}=\frac{\sigma_{t \max }}{\sigma_{t \min }},
$$

where $\sigma_{t \max }$ is the maximum "tensile strength" and $\sigma_{t \min }$ is the minimum "tensile strength". 

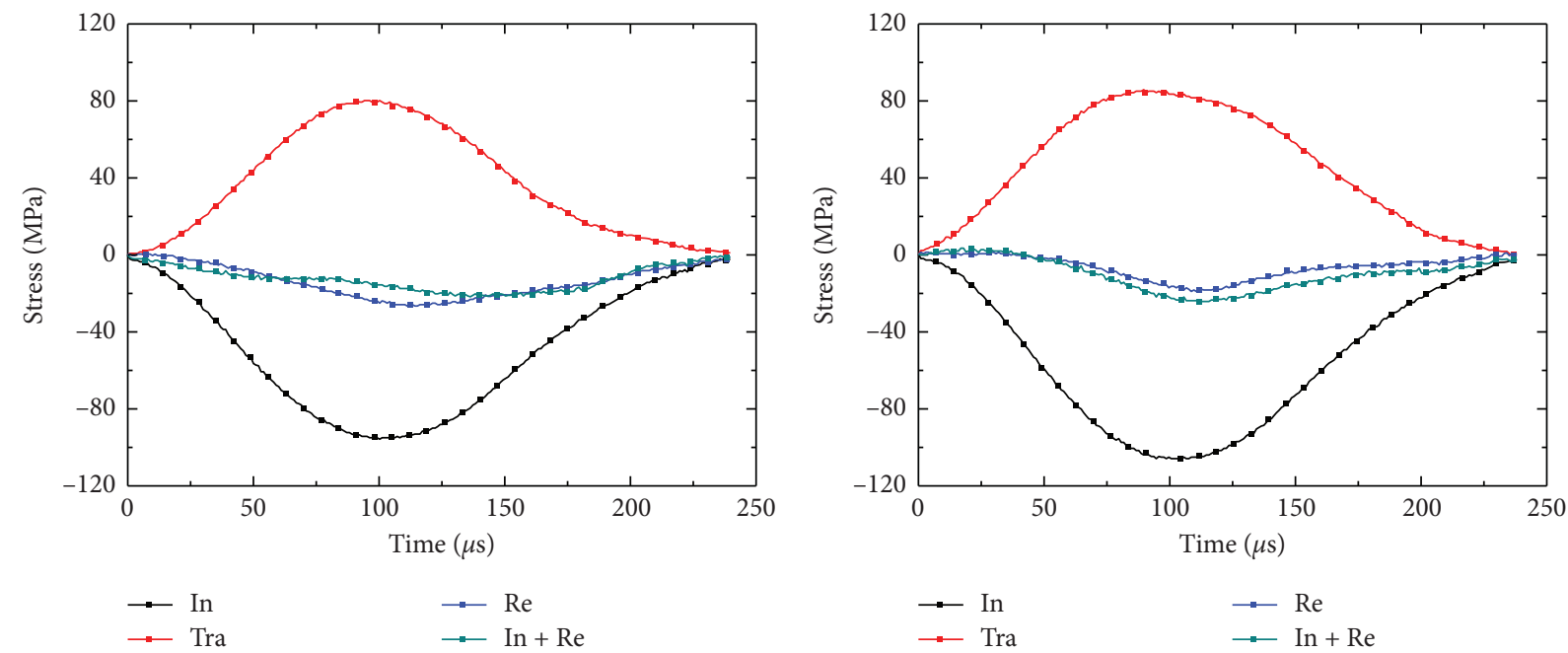

(a)

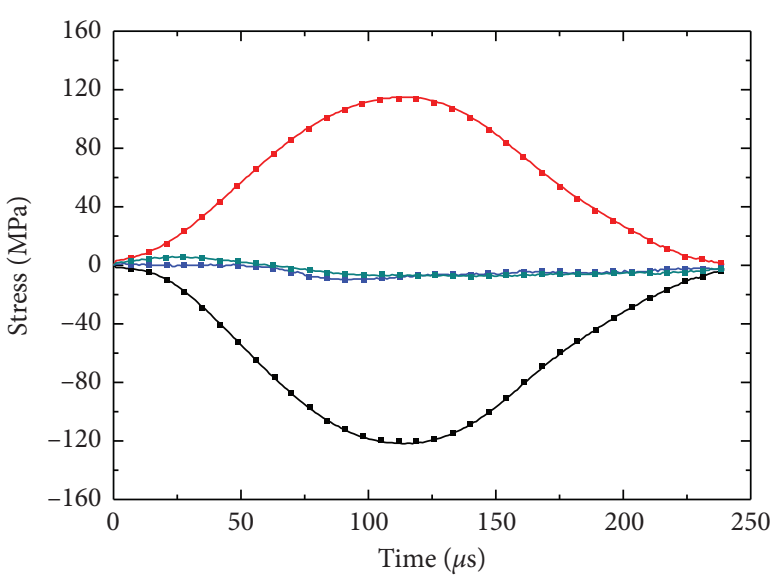

(b)

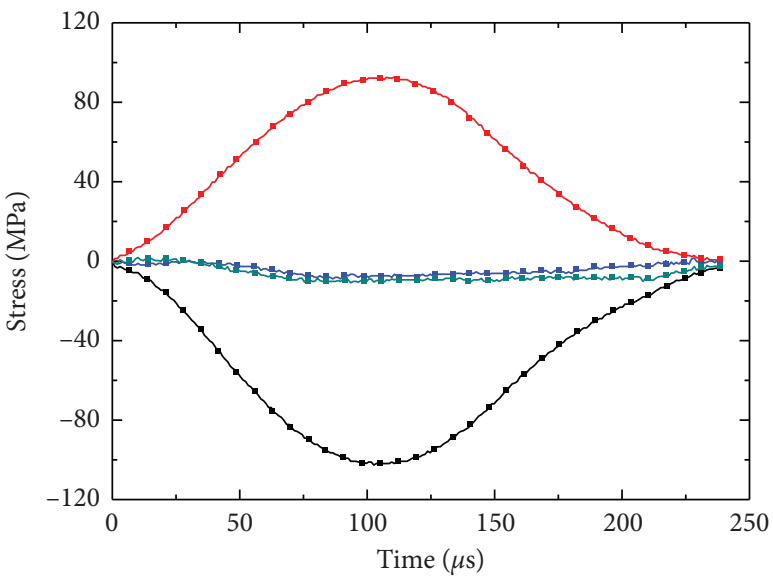

$\begin{array}{ll}\rightarrow & \text { In } \\ \rightarrow & \text { Tra }\end{array}$

$\rightarrow \operatorname{Re}$

$\rightarrow$ In

$\rightarrow \mathrm{Re}$

$\longrightarrow$ In + Re

(c)



(d)

$\because \mathrm{In}+\mathrm{Re}$

$$
\begin{array}{ll}
\rightarrow & \text { In } \\
\rightarrow \operatorname{Tra} & \rightarrow \operatorname{Re} \\
&
\end{array}
$$

(e)

FIGURE 7: Stress equilibrium check for each dip angle. (a) $\theta=0^{\circ}$, (b) $\theta=30^{\circ}$, (c) $\theta=45^{\circ}$, (d) $\theta=60^{\circ}$, and (e) $\theta=90^{\circ}$. 

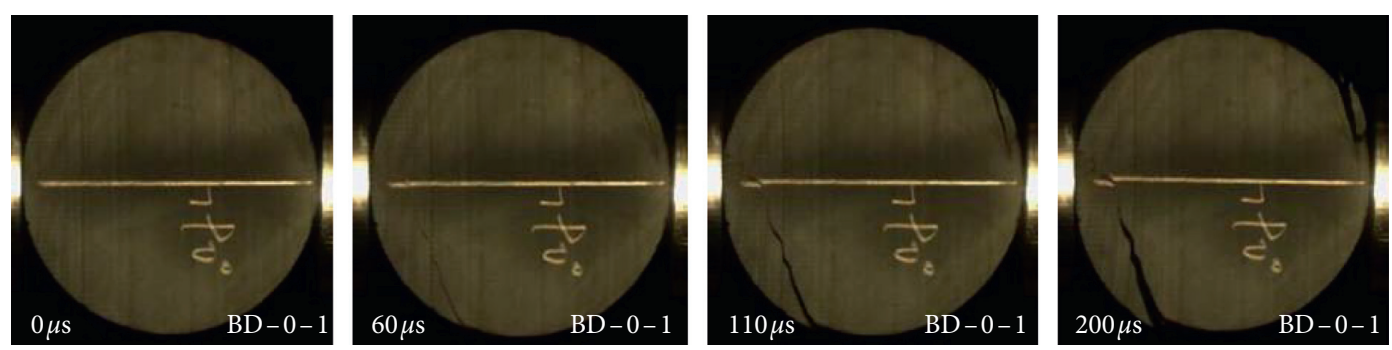

(a)


(b)
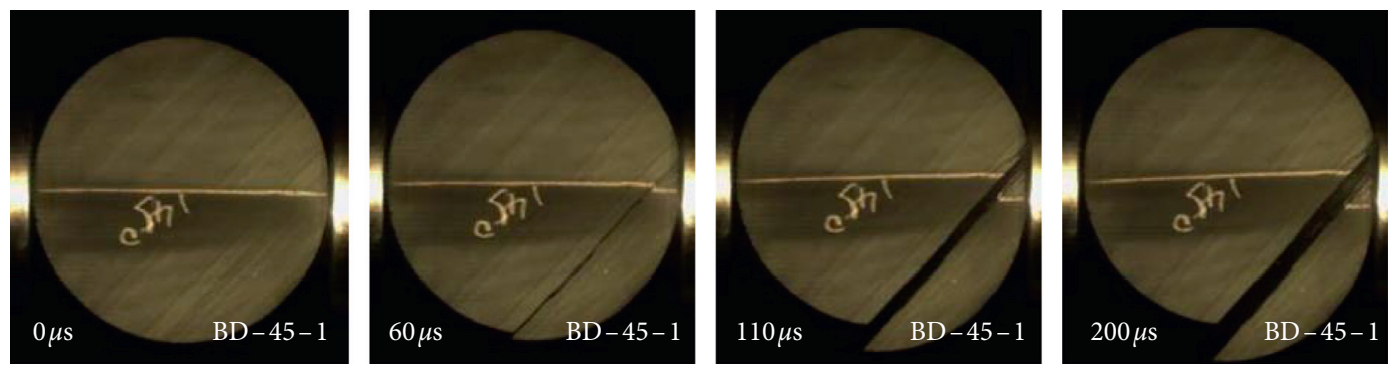

(c)
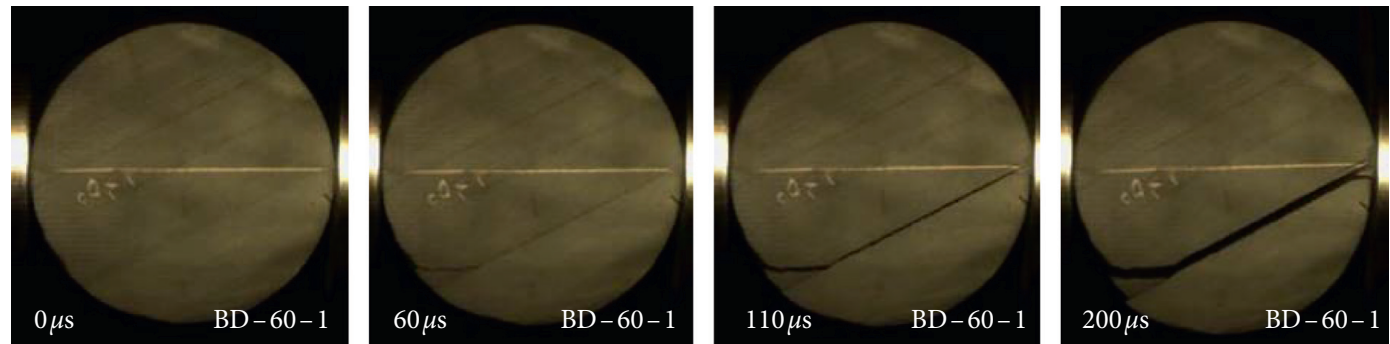

(d)
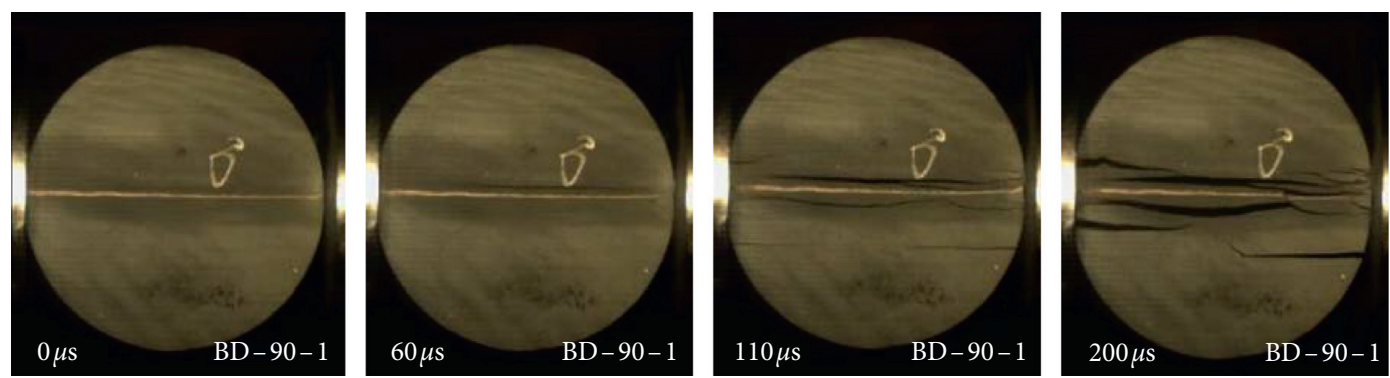

(e)

Figure 8: Typical failure modes of samples with different dip angles. (a) $\theta=0^{\circ}$, (b) $\theta=30^{\circ}$, (c) $\theta=45^{\circ}$, (d) $\theta=60^{\circ}$, and (e) $\theta=90^{\circ}$. 


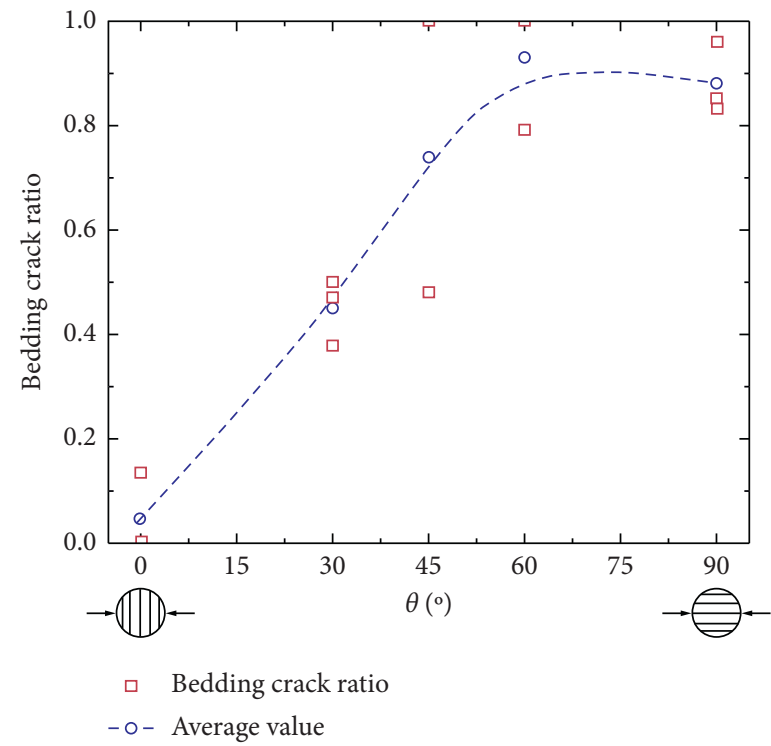

FIgURE 9: Ratio of bedding crack length to total crack length.

TABle 3: Dynamic Brazilian test results of the slate.

\begin{tabular}{|c|c|c|c|c|c|}
\hline$\theta$ & No. & $v_{p}(\mathrm{~km} / \mathrm{s})$ & $\sigma_{t, d}(\mathrm{MPa})$ & Loading rate $(\mathrm{GPa} / \mathrm{s})$ & Energy absorption \\
\hline \multirow{3}{*}{$0^{\circ}$} & $0-1$ & 5.43 & 31.15 & 955 & 11.49 \\
\hline & $0-2$ & 5.52 & 31.01 & 890 & 11.91 \\
\hline & $0-3$ & 5.76 & 27.48 & 936 & 11.79 \\
\hline \multirow{3}{*}{$30^{\circ}$} & $30-1$ & 5.78 & 25.53 & 1117 & 14.64 \\
\hline & $30-2$ & 5.55 & 22.74 & 1034 & 13.18 \\
\hline & $30-3$ & 5.39 & 20.34 & 1030 & 13.20 \\
\hline \multirow{3}{*}{$45^{\circ}$} & $45-1$ & 5.55 & 20.61 & 1050 & 5.96 \\
\hline & $45-2$ & 5.50 & 11.3 & 927 & 5.64 \\
\hline & $45-3$ & 5.33 & 10.81 & 986 & 7.18 \\
\hline \multirow{3}{*}{$60^{\circ}$} & $60-1$ & 5.79 & 14.59 & 979 & 8.05 \\
\hline & $60-2$ & 5.39 & 8.20 & 1012 & 10.61 \\
\hline & $60-3$ & 5.48 & 12.52 & 998 & 8.92 \\
\hline \multirow{3}{*}{$90^{\circ}$} & $90-1$ & 5.39 & 15.96 & 986 & 13.29 \\
\hline & $90-2$ & 5.62 & 14.25 & 1000 & 11.99 \\
\hline & $90-3$ & 5.43 & 14.70 & 1004 & 12.10 \\
\hline
\end{tabular}

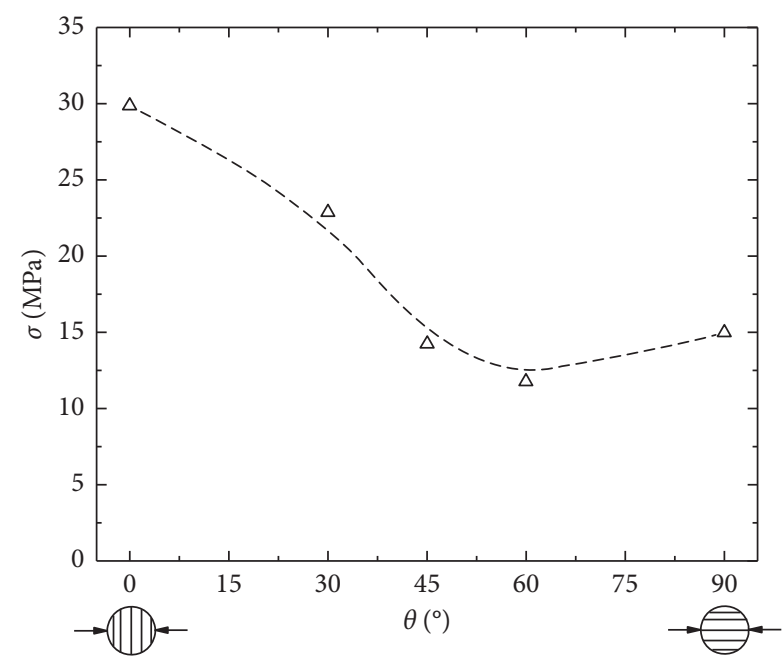

Figure 10: Dynamic "tensile strength" curve of the layered slate under different dip angles.
The "tensile strength" anisotropy measured of this slate is $\alpha_{t}=3.52$. As shown in Figure 11, Tan et al. studied the failure mode of sandstone under different dip angles by the Brazilian test [17]. It can be seen from the figure that the sandstone bedding plane has little influence on its failure mode, and the main crack cracking along the loading direction appears in the samples, which is mainly due to the quasi-isotropic rock $\left(\alpha_{t}=1.03\right)$. Therefore, the failure mode of the transversely isotropic rock under the Brazilian test depends not only on the influence of the bedding plane but also on the anisotropy of the sample. Table 4 statistics some test results of several layered rocks measured by the Brazilian test. It can be concluded that with the increase of anisotropy of splitting "tensile strength", the influence of bedding plane on its failure mode also increases.

6.2. Comparative Analysis of Dynamic and Static Test Results. Figure 12 shows the average value of static/dynamic "tensile strength" under different dip angles. The "tensile strength" 


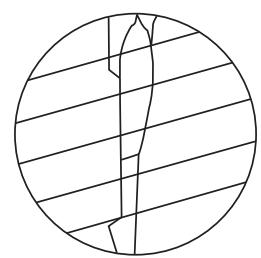

(a)



(b)

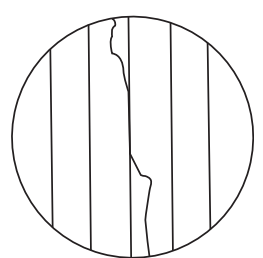

(c)

FIgURE 11: Failure mode of sandstone with dip angles of $15^{\circ}, 45^{\circ}$, and $90^{\circ}$ [10].

TABLE 4: Different rock anisotropy and failure modes.

\begin{tabular}{lcccc}
\hline Types & $\alpha_{t}$ & $\sigma_{t \max } / \theta$ & $\sigma_{t \min } / \theta$ & $\begin{array}{c}\text { Effect of bedding } \\
\text { plane }\end{array}$ \\
\hline Sandstone [17] & 1.22 & $4.14 / 30^{\circ}$ & $3.39 / 0^{\circ}$ & Relatively low \\
Marble [17] & 2.04 & $17.66 / 0^{\circ}$ & $8.67 / 90^{\circ}$ & Low \\
Sandstone [17] & 2.88 & $16.75 / 0^{\circ}$ & $5.80 / 90^{\circ}$ & Medium \\
Slate & 3.52 & $17.24 / 0^{\circ}$ & $4.90 / 45^{\circ}$ & High \\
Slate [17] & 4.09 & $15.56 / 0^{\circ}$ & $3.80 / 75^{\circ}$ & High \\
\hline
\end{tabular}

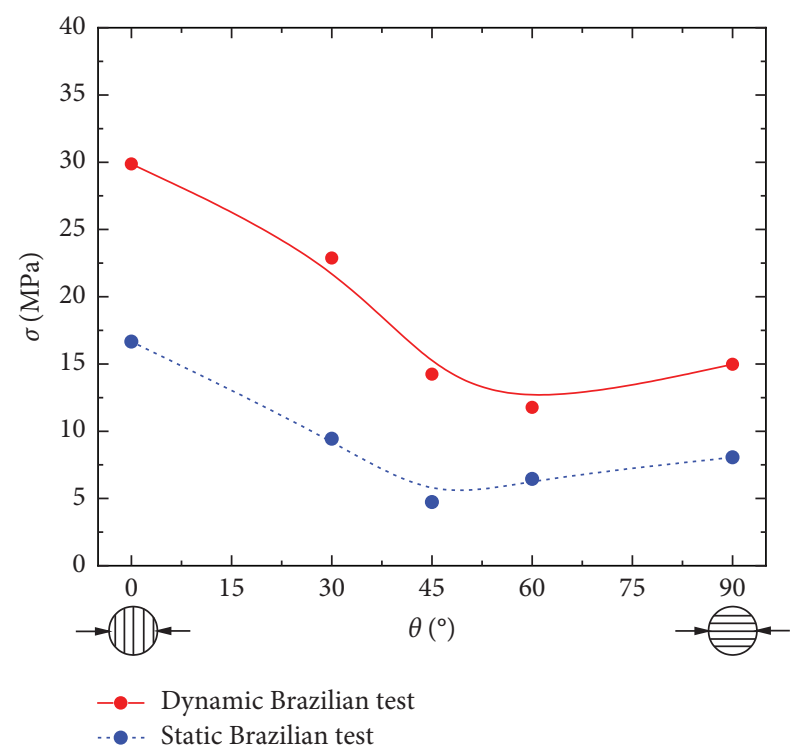

FIGURE 12: Comparison of dynamic and static "tensile strength" under different dip angles.

curve of these two types of tests first decrease and then increase with the increase of the dip angle. Figure 12 also shows that significant strain rate strengthening effect can be observed in Brazilian tests at each dip angle. Although static and dynamic "tensile strength" distribution are both typically " $U$ " type, there are still some differences between the dynamic and static anisotropy curves. The main difference is the minimum "tensile strength" obtained by the static tests is located at $\theta=45^{\circ}$, while the dynamic tests is located at $\theta=60^{\circ}$.

In order to further study the difference between the static and dynamic "tensile strength" of the slate, DIF (dynamic increase factor) values [29] of the test results are calculated. In this study, the factor is the ratio of dynamic and static "tensile strength":
TABLe 5: Dynamic "tensile strength" DIF value of the slate.

\begin{tabular}{ll}
\hline$\theta$ & DIF \\
\hline $0^{\circ}$ & 1.79 \\
$30^{\circ}$ & 2.42 \\
$45^{\circ}$ & 3.01 \\
$60^{\circ}$ & 1.83 \\
$90^{\circ}$ & 1.86 \\
Average & 2.18 \\
\hline
\end{tabular}

$$
\mathrm{DIF}=\frac{\sigma_{t, d}}{\sigma_{t}}
$$

where $\sigma_{t, d}$ is the dynamic "tensile strength" and $\sigma_{t}$ is the static "tensile strength."

The calculated DIF values are listed in table 5. It can be seen from the figure that the strength growth rate of the two tests increases with the increase of bedding dip angle from $\theta=0^{\circ}$ to $\theta=45^{\circ}$ and then decreases rapidly at $\theta=60^{\circ}$. The growth rate of the conventional Brazilian test with $\theta=90^{\circ}$ was the same as that of $\theta=60^{\circ}$ and $\theta=0^{\circ}$. Considering the growth rate of "tensile strength" of the two tests, the dip angle of bedding plane has a significant effect on the growth rate. Under the same loading level, the strength growth of the Brazilian splitting test is the most significant when $\theta=45^{\circ}$ and the slowest at $\theta=60^{\circ}$. Therefore, according to the above conclusions, choosing reasonable impact loading angle in the process of rock drilling and blasting is conducive to improve energy utilization rate and rock breaking efficiency, which is of great significance in underground engineering construction.

\section{Conclusion}

In this study, the static and dynamic tensile properties of the layered slate are studied by Brazilian tests. A medium strength anisotropy slate is cored and tested in five groups of samples with different bedding dip angles. The static and dynamic mechanical properties and failure modes of the slate under different dip angles are obtained.

(1) The failure mode of the static Brazilian test is generally sliding failure along the bedding plane at $\theta=45^{\circ}$ and $\theta=60^{\circ}$. The anisotropy of the slate $\left(\alpha_{t}=3.52\right)$ has clear influence on the failure mode. The failure mode of the layered rock is affected by two factors, the bedding structure and the anisotropy ratio. 
(2) The slate shows significant "tensile strength" anisotropy under the static and dynamic Brazilian tests. The "tensile strength" first decreases and then increases with the increase of the bedding dip angle, which is typically " $U$ " type strength anisotropy. The main difference between the two is that the minimum value appears at the position of the bedding dip angle. The minimum value under static condition is located at $\theta=45^{\circ}$ while in dynamic condition, it is located at $\theta=60^{\circ}$.

(3) The dynamic strengthening effect is significant for samples in the same dip angle. Under the similar loading rate, the orientation of the bedding plane has a significant effect on the "tensile strength" growth, which is most significant when $\theta=45^{\circ}$.

\section{Data Availability}

The experimental data used to support the findings of this study are included within the article.

\section{Conflicts of Interest}

The authors declare that there are no conflicts of interest regarding the publication of this paper.

\section{Acknowledgments}

This research was financially supported by the National Natural Science Foundation of China (no. 52008039), Open Fund of National Engineering Laboratory of Highway Maintenance Technology (Changsha University of Science and Technology), and Scientific research project of Hunan Education Department (no. 19B031).

\section{References}

[1] K. Duan and C. Y. Kwok, "Discrete element modeling of anisotropic rock under Brazilian test conditions," International Journal of Rock Mechanics and Mining Sciences, vol. 78, pp. 46-56, 2015.

[2] Q. Zhang, K. Duan, W. Xiang, Y. Jiao, and S. Yuan, "Direct tensile test on brittle rocks with the newly developed centering apparatus," Geotechnical Testing Journal, vol. 41, no. 1, Article ID 20160301, 2017.

[3] X. Xu, B. Liu, S. Li, J. Song, M. Li, and J. Mei, "The electrical resistivity and acoustic emission response law and damage evolution of limestone in Brazilian split test," Advances in Materials Science and Engineering, vol. 2016, Article ID 8052972, 8 pages, 2016.

[4] F. Gong, W. Wu, T. Li, and X. Si, "Experimental simulation and investigation of spalling failure of rectangular tunnel under different three-dimensional stress states," International Journal of Rock Mechanics and Mining Sciences, vol. 122, Article ID 104081, 2019.

[5] F. Dai and K. Xia, "Loading rate dependence of tensile strength anisotropy of Barre granite," Pure and Applied Geophysics, vol. 167, no. 11, pp. 1419-1432, 2010.

[6] F.-q. Gong, S. Luo, and J.-y. Yan, "Energy storage and dissipation evolution process and characteristics of marble in three tension-type failure tests," Rock Mechanics and Rock Engineering, vol. 51, no. 11, pp. 3613-3624, 2018.

[7] ISRM, "Suggested methods for determining tensile strength of rock materials," International Journal of Rock Mechanics and Mining Sciences and Geomechanics Abstracts, vol. 15, no. 3, pp. 99-103, 1978.

[8] ASTM, D3967-08, Standard Test Method for Splitting Tensile Strength of Intact Rock Core Specimens (Superseded), ASTM International, West Conshohocken, PA, USA, 2008.

[9] T. Ma, N. Peng, Z. Zhu, Q. Zhang, C. Yang, and J. Zhao, "Brazilian tensile strength of anisotropic rocks: review and new insights," Energies, vol. 11, no. 2, p. 304, 2018.

[10] T. Abbass and V. André, "Effect of layer orientation on the failure of layered sandstone under Brazilian test conditions," International Journal of Rock Mechanics and Mining Sciences, vol. 47, no. 2, pp. 313-322, 2010.

[11] G. Khanlari, B. Rafiei, and Y. Abdilor, "An experimental investigation of the Brazilian tensile strength and failure patterns of laminated sandstones," Rock Mechanics and Rock Engineering, vol. 48, no. 2, pp. 843-852, 2015.

[12] Y.-K. Lee and S. Pietruszczak, "Tensile failure criterion for transversely isotropic rocks," International Journal of Rock Mechanics and Mining Sciences, vol. 79, pp. 205-215, 2015.

[13] J. Zhao and H. B. Li, "Experimental determination of dynamic tensile properties of a granite," International Journal of Rock Mechanics and Mining Sciences, vol. 37, no. 5, pp. 861-866, 2000.

[14] M. Cai, P. K. Kaiser, F. Suorineni, and K. Su, "A study on the dynamic behavior of the Meuse/Haute-Marne argillite," Physics \& Chemistry of the Earth, vol. 32, no. 8, pp. 907-916, 2005.

[15] Q. Z. Wang, W. Li, and X. L. Song, "A method for testing dynamic tensile strength and elastic modulus of rock materials using SHPB," Pure and Applied Geophysics, vol. 163, no. 5-6, pp. 1091-1100, 2006.

[16] J. Qiu, D. Li, and X. Li, "Dynamic failure of a phyllite with a low degree of metamorphism under impact Brazilian test," International Journal of Rock Mechanics and Mining Sciences, vol. 94, pp. 10-17, 2017.

[17] X. Tan, H. Konietzky, T. Frühwirt, and D. Q. Dan, "Brazilian tests on transversely isotropic rocks: laboratory testing and numerical simulations," Rock Mechanics and Rock Engineering, vol. 48, no. 4, pp. 1341-1351, 2015.

[18] X. B. Li, T. S. Lok, J. Zhao, and P. J. Zhao, "Oscillation elimination in the Hopkinson bar apparatus and resultant complete dynamic stress-strain curves for rocks," International Journal of Rock Mechanics and Mining Sciences, vol. 37, no. 7, pp. 1055-1060, 2000.

[19] X. B. Li, T. S. Lok, and J. Zhao, "Dynamic characteristics of granite subjected to intermediate loading rate," Rock Mechanics and Rock Engineering, vol. 38, no. 1, pp. 21-39, 2005.

[20] F.-Q. Gong and G.-F. Zhao, "Dynamic indirect tensile strength of sandstone under different loading rates," Rock Mechanics and Rock Engineering, vol. 47, no. 6, pp. 2271-2278, 2014.

[21] X. Ou, X. Zhang, H. Feng, C. Zhang, and J. Yang, "Effect of the confining pressure on the dynamic compression properties of transversely isotropic rocks," Advances in Civil Engineering, vol. 2019, Article ID 5239374, 11 pages, 2019.

[22] F. Dai, K. Xia, J. P. Zuo, R. Zhang, and N. W. Xu, "Static and dynamic flexural strength anisotropy of Barre granite," Rock Mechanics and Rock Engineering, vol. 46, no. 6, pp. 1589-1602, 2013.

[23] F. Gong and J. Hu, "Energy dissipation characteristic of red sandstone in the dynamic Brazilian disc test with SHPB 
setup," Advances in Civil Engineering, vol. 2020, Article ID 7160937, 10 pages, 2020.

[24] X. Li, H. Lai, and D. Gu, "Energy absorption of rock fragmentation under impulsive loads with different waveforms," Transactions of Nonferrous Metals Society of China, vol. 1, pp. 3-7, 2009.

[25] T. Ramamurthy, "Strength and modulus responses of anisotropic rocks," Comprehensive Rock Engineering, vol. 1, no. 13, pp. 313-329, 1993.

[26] K. Xia and W. Yao, "Dynamic rock tests using split Hopkinson (Kolsky) bar system - a review," Journal of Rock Mechanics and Geotechnical Engineering, vol. 7, no. 1, pp. 27-59, 2015.

[27] K. Xia, M. H. B. Nasseri, B. Mohanty, F. Lu, R. Chen, and S. N. Luo, "Effects of microstructures on dynamic compression of Barre granite," International Journal of Rock Mechanics and Mining Sciences, vol. 45, no. 6, pp. 879-887, 2008.

[28] F. Dai and K. W. Xia, "Laboratory measurements of the rate dependence of the fracture toughness anisotropy of Barre granite," International Journal of Rock Mechanics and Mining Sciences, vol. 60, no. 2, pp. 57-65, 2013.

[29] Q. B. Zhang and J. Zhao, "A review of dynamic experimental techniques and mechanical behaviour of rock materials," Rock Mechanics and Rock Engineering, vol. 47, no. 4, pp. 1411-1478, 2014. 